\title{
Prevention of Hypotension following Spinal Anaesthesia in Caesarean Section - then and now
}

\author{
Mitra JK ${ }^{1}$ \\ ${ }^{1}$ Department of Anaesthesia \\ College of Medicine and JNM Hospital \\ Kalyani, Nadia, India
}

\author{
Corresponding Author \\ Jayanta Kumar Mitra \\ Department of Anaesthesia \\ College of Medicine and JNM Hospital \\ Kalyani, Nadia, India \\ Email.mitra.jayanta@gmail.com
}

\begin{abstract}
Hypotension during spinal anaesthesia for caesarean section remains a common scenario in our clinical practice. Certain risk factors play a role in altering the incidence of hypotension. Aortocaval compression counteraction does not help to prevent hypotension. Intravenous crystalloid prehydration has poor efficacy; thus, the focus has changed toward co-hydration and use of colloids. Phenylephrine is established as a firstline vasopressor, although there are limited data from high-risk patients. Ephedrine crosses the placenta more than phenylephrine and cause possible alterations in the foetal physiology.
\end{abstract}

\section{Key Words}

caesarean section, hypotension, spinal anaesthesia

\section{INTRODUCTION}

Spinalanaesthesiahasbecomethemethodofchoicefor anesthesiaforelectivecaesareandelivery. Itisfrequently accompaniedbyhypotension, whichmaybedefinedin absolute terms as a systolic blood pressure of 90 or 100 $\mathrm{mmHg}$ or in relative terms as a percentage (20 percent from baseline). Hypotension caused by a reduction in systemicvascularresistanceisnormallycompensatedby an increase in cardiac output. This is attenuated under spinalanaesthesiabyanincreaseinvenouscapacitance because ofvenodilatationinthelowerpartofthebody. The situation is further compounded in pregnancy by aortocavalcompression.Thus, insteadofcompensatory increase, cardiac output usually decreases. ${ }^{2}$ This is the combinedeffectofreducedcardiacoutputanddecreased systemicvascularresistanceaccountsforhypotensionafter spinal anaesthesia.

\section{ETIOLOGY}

Theincidenceofhypotensioncanbeashighas $80 \%{ }^{3}$; the severitydependsontheheightoftheblock, theposition oftheparturient,andwhetherprophylacticmeasureswere taken to prevent the hypotension.

Measuresthatdecreasetheriskofhypotensiontovarying degrees include intravenous administration of fluids, avoidanceofaortocavalcompression, andmonitoring ofbloodpressureatfrequentintervalsafterplacementof regionalanaesthetic.lfrecognizedandtreatedpromptly, transientmaternalhypotensionmaynotbeassociated with maternal or neonatal morbidity. ${ }^{4}$

The higher the segmental sympathetic blockade, the greateristheriskofhypotensionandassociatedemetic symptoms. ${ }^{5}$ Thesupinepositionsignificantlyincreasesthe incidenceofhypotension. Uelandandcolleaguesobserved an average reduction ofblood pressure from $124 / 72$ to $67 / 38 \mathrm{mmHginmotherswhowereplacedinthesupine}$ position following the induction of spinal anesthesia, whereasthebloodpressureaveraged $100 / 60 \mathrm{mmHgfor}$ mothers in the lateral position. ${ }^{6}$

Uterinebloodflowis pressuredependentasthereisno autoregulationontheplacentalbed.Asaconsequence of this, prolonged maternal hypotension is damaging to the fetus and it is also frequently associated with maternalnauseaandvomiting.Briefepisodesofmaternal hypotensionhaveloweredApgarscores, prolongedtimeto sustained respiration and prolonged fetal acidosis. ${ }^{7}$

\section{AORTOCAVAL COMPRESSION}

Aortocaval compression must be avoided before and during the performance of caesarean section. During supinepositionthegraviduterusofthepregnantwoman compressestheaortaandtheinferiorvenacavaagainst 
thebodiesoflumbarofvertebra.Thisresultsindecreased venous return which may decreases maternal cardiac output and blood pressure leading to compromised uteroplacental perfusion. Therefore, it is necessary to maintain left uterine displacement before and during caesareansection, regardlessoftheanaesthetictechnique. ${ }^{8}$ This may be accomplished by placing a wedge of 12 centimeterbeneath therightbuttock.Althoughwidely used, this procedure is variably applied, ${ }^{9}$ and does not prevent hypotension after spinal anesthesia. ${ }^{10}$

\section{INTRAVENOUS FLUID THERAPY}

Fluidpre-loading wasroutinelyusedupto $87 \%$ ofcasesin spinalanesthesiaforcaesariansection. ${ }^{11}$ Routetalnoted thattheincidenceofhypotensionwasreducedfrom $71 \%$ inpatientswithoutprehydrationto 55\%inpatientswho received crystalloid $20 \mathrm{ml} / \mathrm{kg}^{12}$ However, some study showedthatusing $10 \mathrm{ml}-30 \mathrm{ml} / \mathrm{kg}$ Ringer'slactateforacute volumeexpansionbeforeinduction ofspinalanesthesia, no differences in the indices of maternal hypotension ordosage of ephedrinewasobserved. ${ }^{13}$ Both the rate ${ }^{14}$ and volume ${ }^{15}$ of crystalloid preloading have also been showntobeunimportant.Studiesofthiskind haveledto a reappraisal of the role of fluid preloading. ${ }^{16,17}$ It is still reasonabletoadministeramodestamountofcrystalloid preload before spinal injection, as patients for elective surgeryareoftenrelativelydehydrated. However,thereis noneedtodelayemergencysurgeryinordertopreload.

A recent systematic review found that crystalloid was inconsistentinpreventinghypotensionandthatcolloidwas significantlybetter. ${ }^{18}$ Dahlgrenetal ${ }^{19}$ studiedcrystalloid comparedwithcolloidforpreloading. Hypotensionwas significantlyreducedafterlargervolumes ofcolloid.Itis postulatedthatparturientpreoperativelysusceptibleto thesupinepositionwould benefitthemostfromcolloid preloading. In anotherstudy of preloading comparing pentastarchwithcrystalloid,Frenchetal ${ }^{20}$ demonstrated areductionintheincidenceofhypotensioninthecolloid group(12.5\%versus $47.5 \%)$.Incontrasttothesestudies which all found colloid preload of benefit, Karinen et al failedtofindanyreductionintheincidenceofhypotension when colloid was used. ${ }^{21}$ Moreover, disadvantages of colloid include the additional cost, possibility of anaphylactoidreactionsandexcessivevolumeexpansion, which might lead to pulmonary oedema. ${ }^{22}$

Several recent studies have compared prehydration versus cohydration both with crystalloids and colloids andshownthathaemodynamicchangesandvasopressor requirementaresimilar.Banerjeeetal performedametaanalysis (eight studies, 518 partuients) of studies that comparedprehydrationwithcohydration. Theyfoundthat theincidenceofhypotensiontobesimilarfor(oddsratio $0.93,95 \%$ confidenceinterval[Cl]0.54-1.6)cohydration to that for prehydration. ${ }^{23}$

To sum up, firstly, colloid is superior to crystalloid for fluidmanagementwithsomerecognizableadverseeffects; secondly, one should consider the role of vasopressor alongwiththefluidusedinmanagementofhypotension ${ }^{24}$; andthirdly, prehydrationisnotsuperiortocohydration, implyingthatanyurgentcaseshouldnotbedelayedonthe pretext of prehydration.

\section{VASOPRESSORS}

Ephedrinehasbeenthedrug ofchoiceformorethan 30 yearsinthetreatmentofmaternalhypotensioninobstetric spinalanesthesiawhenconservativemeasuresfail.Ithas agoodsafetyrecord, readyavailability, andfamiliarityto mostanesthesiologists.Ephedrineisasympathomimetic thathasbothadirect(alphaandbetareceptoragonist)and anindirect(releaseofnorepinephrinefrompresynaptic nerveterminals)mechanismofaction.Uterinebloodflow, in particularwasmaintainedmorefavorablywith betaagoniststhanwithalpha-agonists.Ephedrinethusbecame thegoldstandardforthisapplicationand,in2001, asurvey ofobstetricanesthetistsintheUnitedKingdomfoundthat morethan $95 \%$ usedephedrineasthesolevasopressor, withonly $0.4 \%$ choosingphenylephrine. ${ }^{11}$ Ephedrinehas aslowonsetofactionmaking itdifficulttotitrateanduse itwithanappropriatebolusdose.Regardingephedrine prophylaxis,studieshavelookedattheeffectivenessto preventmaternalhypotension.NganKeeandcolleagues found that a 30 - mg bolus of ephedrine administered over30secondsfollowing intrathecal injection did not completely eliminate maternal hypotension, nausea, vomitingandfetalacidosis. ${ }^{25}$ Shearerandcolleaguesalso havefoundsimilarresult.Thus, asingleprophylacticdoseis ineffectiveandtheeffectivenessdependsonvariabledoses andtherateofadministration. ${ }^{26}$ Thereasonwhyephedrine depressesfetalacid-basestatusmorethanphenylephrine is controversial. Older studies focused on differential effects of vasopressors on uteroplacentral circulation. However, Ngan Knee et a ${ }^{27}$ showed that ephedrine crossestheplacentamore readily than phenylephrine. Thiswasassociated with greaterfetal concentrations of lactate, glucoseandcatecholamine, and thussupports thehypothesisthatdepressionoffetalpHandmetabolic effectssecondarytostimulationoffetalbeta-adrenergic receptorscausebaseexcesswithephedrine.Ephedrine, withitslongduration ofactionstillhasaroleinobstetric anesthesiatopreventortreatspinalinducedhypotension whengiveninanappropriatedose.Theoptimalmethod toadministerephedrine, whethercombined withother 
vasopressortherapyornonmedicationtherapy, awaits future study.

Phenylephrineisashort-acting,potent, vasoconstrictor thatcausesanincreaseinbothsystolicanddiastolicblood pressure.Itcounteractsthevasodilatationduetoneuraxial anaesthesiadirectly, restoring baselineblood pressure. Traditionally, itwasusedasasecondlinevasoconstrictor in obstetrics because of the concerns that it caused vasoconstrictionintheuteroplacentalcirculation.Interest in phenylephrinewasrekindledin 1988by Ramanathan and Grant, ${ }^{28}$ whofound thatitdidnotcausefetalacidosis whentreatingmaternalhypotension.Numerousstudies haveconfirmedthesefindingsandalmostallhavereported higher umbilical artery(UA) $\mathrm{pH}$ values in neonates borntophenylephrinetreatedmothers. ${ }^{29} \mathrm{~A}$ systematic review in 2002 summarized findings from seven RCTs comparingephedrinewithphenylephrine. ${ }^{30}$ Inthisreview phenylephrinewasassociated withhigherUApHvalues thanephedrinealthoughtherewasnodifferenceinthe incidence of fetal acidosis ( $\mathrm{UA} \mathrm{pH}<7.2)$ or in the Apgar scores $<7$ at 1 and 5 minutes. Whenthereishypotension and bradycardiaephedrinecontinuestobethedrug of choice $^{31}$.Otherwise, phenylephrine, which hasnotbeen showntobedeleterioustothefetus, maywellbethebetter agent.Therearelimiteddatacomparingephedrineand phenylephrinewithregardtoothermaternaloutcomesof interestincludingnauseaandvomiting.Onestudyfound that the incidence of nausea was $66 \%$

in ephedrine treated mothers compared with $17 \%$ in the phenylephrine group. ${ }^{32} \mathrm{~A}$ recent randomized clinicaltrialexaminedthematernalandneonataleffects of maintaining maternal blood pressure within $80 \%$, $90 \%$, or $100 \%$ of baseline levels using a phenylephrine infusion..$^{33}$ Using phenylephrine $100 \mathrm{mcg} / \mathrm{mlinfusedat}$ initial rates of $100 \mathrm{mcg} / \mathrm{min}$, theinvestigatorsadjusted thedosedependinguponwhetherblood pressurewas kept within the assigned group's range. Woman in the 100\%baselinegrouphadfewerepisodes ofnauseaand vomitingandtheirneonatalmeanumbilicalarterial $\mathrm{pH}$ washigher.Hypotensionwasbettercontrolled withtight controlofbloodpressureusingaggressivevasopressor administration.Phenylephrineappearstohavesurvived theperiod ofintensesuspicionand concernoveritsuse inobstetricanesthesia.Itisreliableinitseffect, although shortacting, anditseffectonthefetusappearstobeeven less than that of ephedrine.

Combinations of phenylephrine and ephedrine given together in the same syringe have previously been advocated,althoughtheoptimal regimenhasnotbeen determined. Mercier and colleagues compared an ephedrine/phenylephrineinfusion withanephedrine infusionaloneandfoundthattheincidenceofhypotension inthecombinationgroupwashalfthatintheephedrinealonegroup with a beneficial effecton umbilicalartery $\mathrm{pH}^{34}$ However, whenCooperandcolleaguesperformed arandomized,doubleblind trialcomparingephedrine, phenylephrineandephedrine/phenylepherineinfusions, therewasnodecreaseintheincidenceofmaternalnausea andvomitingorneonatalacidosiswhenthecombination wasusedcomparedwithphenylephrinealone. ${ }^{32}$ Reflecting uponthesestudies, theadministrationofvasopressordrugs byinfusionasclosetothetimeofthespinalanaesthesia administrationaspossibleappearstobehelpfulinreducing the incidence of hypotension.

Metaraminol,amixedalphaandbetaagonistcanbeused forspinalinducedhypotension.NganKeeandcolleagues demonstratedthatmetaraminolwassuperiortoephedrine atmaintainingbothmaternalbloodpressureandfetalpH duringspinalanesthesiaforcaesareansection.Thedoses ofvasoconstrictorsinthisstudywerelargeandthebenefits mayhavebeenexaggerated. ${ }^{35}$ Angiotensinllisapotent vasoconstrictor with a short half life, which affects the uterinevasculaturelessthantheothervasoconstrictors. Raminandcolleaguesdemonstratedabenefittousing angiotensinlloverephedrinewhencomparingfetal $\mathrm{pH}$ after prophylactic infusions of two drugs at caesarean section. ${ }^{36}$ Angotensin ll had to beused in infusion more overotherlimitationsincludesvailabilytandcost.There are only few studies comparing angiotension infusion there is no meta analysis as such.

\section{OTHER METHODS}

Low dose spinal anaesthesia for caesarean delivery combines a small dose of intrathecal local anesthetic with an opiod to reduce the incidence of hypotension. Tsenetalshowedthatwith 12 mgbupivacainealongwith 1000 mloflactatedRinger'ssolutionpreloadingand $10 \mathrm{mg}$ ephedrine,theincidenceofhypotension was $70 \%{ }^{37}$; which furtherloweredto $58 \%$ when 9 mgbupivacainewasused along with 1000 mllactatedRinger'ssolutionpreload with $15 \mathrm{mg}$ ephedrine..$^{38}$ The incidence was further reduced to $31 \%$ when $25 \mu$ goffentanyland 5 mg bupivacainewas used. ${ }^{39}$ Notasinglepatientinthelowdosegroupachieved acompletemotorblock, whereasmostofthepatientsin theplainbupivacainegroupdid.Despitethedifferences inmotorblock,thesensoryblockwassufficientlyintense in both groups to provide surgical anaesthesia for all patients.Althoughthetechniqueispromising, andone mightintuitivelyexpectareduction in theincidence of hypotensionandnauseawithsuchlowdoses, thereare insufficient data to support this conclusion. 


\section{CONCLUSION}

Managementofhypotensionduringspinalanaesthesiain obstetricscontinuestobecontroversial.Althoughmost clinicians will continue to rely on non-invasive BP and cardiac output monitoring may prove useful in future. Whilefluidpreloadandleftuterinedisplacementareoften employedinanattempttopreventthiscomplication, a vasopressorisoften required.Crystalloidprehydration shouldnolongerbeconsideredmandatoryandthecurrent focusisontiming offluidsanduseofcolloids. Apartfrom this, one may choose ephedrine or phenylephrine as a vasopressor.Ephedrinecausesmoredepressionoffetal acid-basestatusthanphenylephrine, probablybecause ephedrinecrossestheplacentamorereadilyandhasdirect metaboliceffectsonthefetus. Thereisanabundance of evidencetosuggestthatphenylephrineisatleastasgood asephedrineandamoreliberaluseofthisdrugisprobably justified.Furtherworkisrequired todeterminetheoptimal therapy for hypotension in high-risk patients.

\section{REFERENCES}

1. Shibli KU, Russell IF. A survey of anaesthetic techniques used for caesarean section in the UK in 1997. Int J Obstet Anesth 2000;9:160-167.

2. Thomas DG, Robson SC, Redfern N, et al. Randomized trialofbolusphenylephrineorephedrineformaintenance ofarterialpressureduringspinalanesthesiaforcaesarean section. Br J Anaesth 1996;76:61-5.

3. LevinA,DattaS,SegalS.Theeffectofpostureonhypotension afterspinalanaesthesiaforcaeseriansection.Anesthesiology 1998;88:A10.

4. Norris MC. Hypotension during spinal anesthesia for caesareansection:Doesitaffectneonataloutcome?Reg Anaesth 1987;12:191-3.

5. HolmesF.Thesupinehypotensivesyndrome.Anaesthesia 1995; 50:972-7.

6. UelandK,GillsRE, andHansenJM:Maternalcardiovascular dynamics.1.Caesareansectionundersubarachnoidblock anaesthesia. Am J Obstet Gynecol 1968;100:42.

7. Reynolds F, Seed PT. Anaesthesia for Caesarean section andneonatalacid-basestatus:ameta-analysis.Anaesthesia. 2005;60:636-53.

8. Milsoml,ForssmanL,BiberB,etal.Maternalhaemodynamic changes during caesarean section: A comparison of epiduralandgeneralanaesthesia.ActaAnaesthesiolScand 1985;29:161-7.

9. Paech MJ. Should we take a different angle in managing pregnantwomenatdelivery?Attemptingtoavoidthe'supine hypotensivesyndrome'.AnaesthIntensiveCare2008;36:7757.

10. Cyna AM, Andrew M, Emmett RS, Middleton $P$, Simmons SW. Techniques for preventing hypotension duringspinalanaesthesiaforcaesareansection.Cochrane Database Syst Rev 2006. Cochrane Database Syst Rev. 2002;(3):CD002251.

11. Burns SM, Cowan CM, Wilkes RG. Prevention and managementofhypotensionduringspinalanaesthesiafor electiveCaesareansection:asurveyofpractice.Anaesthesia 2001;56:794-98.

12. Rout CC, Rocke DA, Levin J, et al. A re-evaluation of the roleofcrystalloidpreloadinthepreventionofhypotension associated withspinalanaesthesiaforelectivecaesarean section. Anesthesiology 1993;79:262-69.

13. Park GE, Hauch MA, Curlin F, Datta S, Bader A: Effects ofvarying volume preload beforeCeasarean deliveryon maternalhaemodynamicsandcolloidosmoticpressure. Anaesth Analg 1996;83:299.

14. Rout CC, Akoojee SS, Rocke DA, Gouws E. Rapid administrationofcrystalloidpreloaddoesnotdecreasethe incidenceofhypotensionafterspinalanaesthesiaforelective caesarean section. Br J Anaesth 1992;68:394-7. 
15. McKinlayJ,LyonsG.Obstetricneuraxialanaesthesia:which pressoragentsshouldwebeusing?IntJObstetAnesth2002; 11:117-21.

16. Riley ET. Spinal anesthesia for Caesarean delivery: keep the pressure upand don'tspare the vasoconstrictors. Br J Anaesth 2004;92:459-61.

17. MorganPJ,HalpernSH,TarshisJ.Theeffectsofanincrease of central blood volume before spinal anesthesia for caesareandelivery:aqualitativesystematicreview.Anaesth Analg 2001;92:997-1005.

18. Dahlgren G, Granath F, Wessel H, Irestedt L. Prediction of hypotensionduringspinalanesthesiaforCesareansection anditsrelationtotheeffectofcrystalloidorcolloidpreload. Int J Obstet Anesth 2007;16:128-34.

19. RileyET,CohenSE, RubensteinAJ,FlanaganB.Prevention ofhypotensionafterspinalanaesthesiaforcaesareansection: $6 \%$ hetastarch versuslactated Ringer'ssolution. Anaesth Analg 1995;81:838-42.

20. FrenchGWG,WhiteJB,HowellSJ,PopatM.Comparisonof pentastarchandHartmann'ssolutionforvolumepreloading in spinal anaesthesia for elective Caesarean section. $\mathrm{Br}$ J Anaesth 1999;83:475-7.

21. Karinen J, Rasanen J, Alahuhta S, Jouppila R, Jouppila P. Effectofcrystalloidandcolloidpreloadingonuteroplacental andmaternalhaemodynamicstateduringspinalanaesthesia for Caesarean section. Br J Anaesth 1995;75:531-35.

22. Weeks S. Reflections on hypotension during caesarean sectionunderspinalanesthesia:doweneed tousecolloid? Can J Anaesth 2000;47:607- 10.

23. Banerjee A,Stocche RM,Angle P,Halpem SH.Preload or coloadforspinalanesthesiaforelectivecesareandelivery:a meta-analysis. Can J Anaesth 2010;57:24-31.

24. NganKeeWD,KhawKS,NgFF.Prevention ofhypotension duringspinalanesthesiaforCesareandelivery:Aneffective techniqueusingcombinationphenylephrineinfusionand crystaloidcohydration.Anesthesiology2005;103:744-50.

25. Ngan Kee WD, Khaw KS,Ng FF. Comparison of phenylephrineinfusionregimensformaintainingmaternal blood pressure during spinal anaesthesia for caesarean section. Br J Anaesth 2004;92:469-74.

26. Shearer VE, Ramin SM, Wallace DH, et al.Fetal effects of prophylacticephedrineandmaternalhypotensionduring regionalanesthesiaforCS.JMaternFetalMed 1996;5:79-84.

27. Ngan Kee WD, Khaw KS, Tan PE, et al. Placentral transferandfetalmetaboliceffectsofphenylephrineand ephedrineduringspinalanaesthesiaforcesareandelivery. Anaesthesiology 2009;111:506-12.

28. Hall PA, Bennett A, Wilkes MP, etal.Spinal anaesthesiafor caesariansection:comparisonofinfusionsofphenylephrine and ephedrine. Br J Anaesth 1994;73:471-74.
29. LaPorta RF, ArthurGR, Datta S.Phenylephrine in treating maternalhypotensionduetospinalanaesthesiaforcaesarean delivery:effectsonneonatalcatecholamineconcentrations, acidbasestatusandApgarscores.ActaAnaesthesiolScand 1995;39:901-5.

30. LeeA,NganKW,GinT.Aquantitative, systematicreviewof randomizedcontroltrialsofephedrineversusphenylephrine forthemanagementofhyptensionduringspinalanesthesia for caesarean delivery. Anesth Analg 2002;94:920-6.

31. Vallejo MC, Ramanathan S. Should alpha-agonists beed asfirstlinemanagementofspinalhypotension?IntJObstet Anesth 2003;12:243-45.

32. Cooper DW, Carpenter M, Mowbray P, et al. Fetal and maternaleffectsofphenylephrineandephedrineduring spinalanesthesiaforcaesareandelivery.Anesthesiology 2002;97:1582-90.

33. NganKeeWD,KhawKS,NgFF.Prophylacticphenylephrine infusionforpreventinghypotensionduringspinalanesthesia for cesarean delivery. Anesth Analg 2004;98:815-21.

34. MercierFJ,RileyET,FredericksenWL,etal.Phenylephrine added to prophylactic ephedrine infusion during spinal anesthesiaforelectiveCS.Anesthesiology2001;95:668-74.

35. Ngan Kee WD, Lau TK, Khaw KS, et al. Comparison of metaraminol and ephedrine infusions for maintaining arterialpressureduringspinalanaesthesiaforelectiveCS. Anesthesiology 2001;95:307-13.

36. Ramin SM, Ramin KD, Cox K, et al. Comparison of prophylacticangiotensinllversusephedrineinfusionfor preventionofmaternalhypotensionduringspinalanesthesia. IS J Obstet Gynecol 1994; 171: 734-9.

37. Tsen LC, Boosalis P, Datta S, Bader AM. Haemodynamic effects of simultaneous administration of intravenous ephedrineandspinalanaesthesiaforcaesareandelivery.J Clin Anesth 2000;12:378-82.

38. Sarvela PJ, Halonen, Korttila.Comparison of $9 \mathrm{mg}$ of intrathecal plain and hyperbaric bupivacaine both with fentanyl for caesarean delivery. Anaesth Analg 1999;89: 1257-62.

39. Ben-David B, Miller G,Gavriel R, Gurevitch A. Low-dose bupivacaine-fentanylspinalanesthesiaforcesareandelivery. Reg Anesth Pain Med 2000;25:235-9. 